\title{
Prof. Dr. Cyril A. Grob zum Gedenken
}

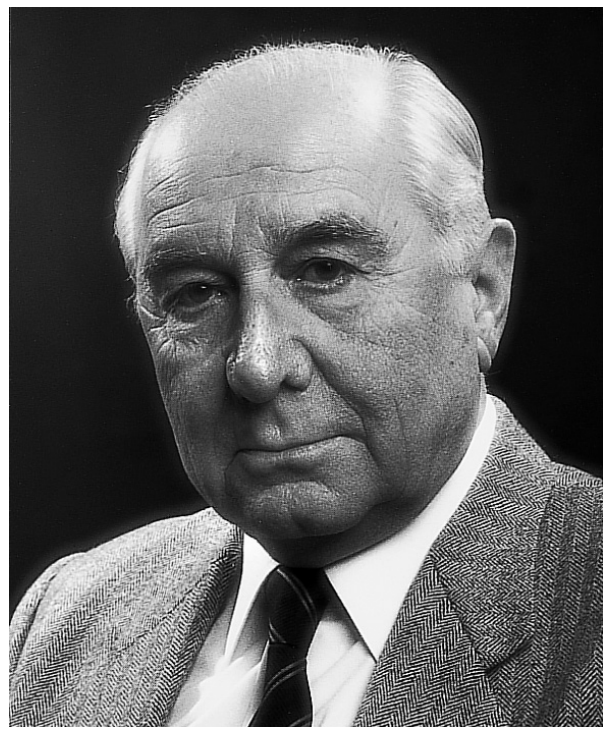

Im Dezember des vergangenen Jahres ist Prof. Cyril A. Grob in seinem Heim in Basel im 87. Lebensjahr verstorben. Cyril Grob hat während des Zweiten Weltkrieges an der Eidgenössischen Technischen Hochschule in Zürich Chemie studiert und im Jahre 1942 bei Prof. L. Ruzicka promoviert. Nach dem Abschluss seines Studiums zog Cyril Grob nach Basel, wo er als Mitarbeiter von Prof. T. Reichstein zunächst am Pharmazeutischen Institut und ab 1947 am Organisch Chemischen Institut der Universität Basel tätig war. Im gleichen Jahr 1947 erfolgte seine Habilitation und 1951, nach einem Studienaufenthalt in den USA, seine Beförderung zum ausserordentlichen Professor. Im Jahre 1954 wurde er zum persönlichen Ordinarius ernannt und 1960, nach dem Rücktritt von Prof. T. Reichstein, erfolgte seine Wahl zum Inhaber des gesetzlichen Lehrstuhls und zum Vorsteher des Instituts für Organische Chemie. Diese Funktion hatte er bis zu seiner Emeritierung im Jahre 1987 inne gehabt.

Als Absolvent der Zürcher Schule befasste sich Cyril Grob am Anfang seiner akademischen Karriere überwiegend mit Problemen aus der Naturstoffchemie. Synthetische Studien am Biotin, an der Lysergsäure und am Steroidgerüst, aber auch eine elegante Synthese von Sphingosin waren Gegenstand seiner frühen Arbeiten in Basel. Die damals verfügbaren Methoden zum Aufbau komplexer Kohlenstoffgerüste und zur Umwandlung funktioneller Gruppen waren nach heutiger Sicht eher rudimentär. Das mag ein Grund dafür gewesen sein, dass sich Cyril Grob in der Folge so intensiv um ein besseres Verständnis für den genauen Ablauf chemischer Umsetzungen und für die Natur der dabei auftretenden kurzlebigen Zwischenprodukte bemüht hat. In dieser Hinsicht erhielt er entscheidende Impulse anlässlich des erwähnten Studienaufenthaltes an der UCLA bei Prof. Saul Winstein im Jahre 1950/51. Der Aufenthalt in Los Angeles hat Cyril Grob nicht nur wertvolle Erfahrungen und wissenschaftliche Anregungen gebracht, sondern auch viele bleibende persönliche Freundschaften vermittelt.

In der Folge hat er sich neben den synthetischen Arbeiten aus der Naturstoffchemie immer mehr mit Fragen des $\mathrm{Zu}$ sammenhanges zwischen Struktur und Reaktivität organischer Verbindungen befasst mit dem Ziel, den Reaktionsmechanismus zentraler Grundreaktionen der synthetischen organischen Chemie zu ergründen. Nachfolgend seien zwei ausgewählte Bereiche aus dem vielfältigen Arbeitsgebiet von Cyril Grob kurz in Erinnerung gerufen:

Oft führen unerwartete Ergebnisse zu neuer Erkenntnis. So hat eine Untersuchung über die reduktive Elimination von Brom mit Zink im Jahre 1955 gezeigt, dass gewisse 1,4-Dibromide unter Spaltung einer $\mathrm{C}, \mathrm{C}$-Bindung und Bildung zweier neuer C,C-Doppelbindungen abreagieren. Diese Beobachtung führte zum Erkennen der heterolytischen Fragmentierung als allgemeinem Reaktionsprinzip in der organischen Chemie. Viele Mitarbeiter haben in der Folge an einer grossen Zahl unterschiedlicher Modellverbindungen die stereochemischen Voraussetzungen für das Eintreten einer Fragmentierungsreaktion untersucht und die verschiedenen Grundmechanismen dieses Reaktionstyps aufgeklärt. Die heterolytische Fragmentierungsreaktion hat heute als eigenständiges Reaktionsprinzip Eingang in die Lehrbücher der organischen Chemie gefunden und wird dort häufig mit dem Namen ihres Entdeckers bezeichnet.

Das Studium des induktiven Effektes hat Cyril Grob über viele Jahre hinweg immer wieder beschäftigt. Er erkannte, dass in 4-Stellung substituierte Chinuklidine geeignete Modellverbindungen darstellen zur Bestimmung klar definierter induktiver Substituentenkonstanten, denn sie tragen den Substituenten in genau bekanntem Abstand vom basischen Stickstoff, der nach Protonierung eine positive Ladung trägt.

Kohlenstoff-Kationen sind als reaktive Zwischenprodukte bei solvolytischen Reaktionen gut dokumentiert. In vielen Fällen reagieren diese unter Umlagerung des Kohlenstoffskeletts, wobei Ladung an benachbarte Zentren im Molekül verschoben wird. Dabei wird eine nichtklassische Zwischenstufe mit fünfbindigem Kohlenstoff durchlaufen. Die Frage, ob eine Ladungsverschiebung bereits vor vollständiger Ablösung der Fluchtgruppe einsetzt oder erst in einem nachfolgenden Elementarprozess erfolgt, war lange Zeit hoch kontrovers. Cyril Grob sah eine Antwort auf diese Frage in 
der Bestimmung der sogenannten Induktivität, das heisst in der Abhängigkeit der Geschwindigkeit des Ionisationsprozesses von Substituenten in Nachbarschaft des reaktiven Zentrums durch ihren individuellen induktiven Substituenteneffekt. Das Ergebnis einer sehr sorgfältig geplanten und umfangreichen kinetischen Studie ergab keine einfache Aussage, sondern zeigte, dass es fliessende Übergänge gibt. Nur in Ausnahmefällen, wie im notorischen Norbornylkation scheint die nichtklassische Struktur stabiler zu sein als eine solche mit lokalisierter Ladung. Das Ergebnis dieser Studie ist 1983 in einer denkwürdigen Publikation in Accounts of Chemical Research den entsprechenden Berichten von H.C. Brown und G.A. Olah gegenübergestellt worden, welche die gleiche Frage je aus einer ganz anderen Richtung mit ähnlichem Ergebnis angegangen waren. Wie der als unabhängiger Schiedsrichter aufgebotene Ch. Walling zusammenfassend feststellen konnte, ist damit die lang und intensiv diskutierte Kontroverse über die Struktur des Norbornylkations friedlich beigelegt worden.

Cyril Grob war eher von zurückhaltender Natur. Er hat sich nie in die Öffentlichkeit gedrängt, hat aber die ihm dank seiner Stellung zugefallenen repräsentativen
Pflichten stets effizient und ohne viel Aufheben erfüllt. In den frühen Sechzigerjahren hat er sich in verdienstvoller Weise um eine Reform des Chemiestudiums in Basel eingesetzt mit klarer Strukturierung des Studienganges und einer Unterteilung der Studienabschnitte durch Vordiplom und Diplomprüfung. Wohl weniger aus Freude am ehrenvollen Amt als vielmehr in Pflichterfüllung hat er die Geschäfte der Naturwissenschaftlichen Fakultät während eines Jahres als Dekan geleitet. Auch der Schweizerischen Chemischen Gesellschaft hat er sich während einer zweijährigen Amtszeit als Präsident zur Verfügung gestellt.

Mit der Aufnahme reaktionsmechanistischer Studien hat Cyril Grob für den Bereich der physikalisch-organischen Chemie in unserem Lande eine Vorreiterrolle übernommen. Davon haben viele Studentengenerationen, aber auch Fachkollegen im Berufsumfeld profitiert. Als Lehrer und Vortragender hat er es verstanden, komplexe Zusammenhänge einfach und klar darzustellen. Durch seinen präzisen und schnörkellosen Vortrag in seinen Vorlesungen und durch sachliche, wohlüberlegte Argumentation im Gespräch hat er nicht nur seine Zuhörer überzeugt, sondern auch immer wieder Studierende dazu motiviert, das Abenteuer einer Dissertation mit ungewissem Ausgang aus seinem Forschungsbereich einzugehen. Er hat seine Mitarbeiterinnen und Mitarbeiter lose geführt und ihnen viele Freiheiten gelassen, ist ihnen aber im richtigen Zeitpunkt mit kompetentem Rat stets hilfreich beigestanden.

Im Laufe der Jahre hat Cyril Grob über hundert Studierende zum Doktorat geführt. Dazu kommt eine grössere Zahl von promovierten Mitarbeitern aus der Schweiz und aus der ganzen Welt. Die Ergebnisse der von ihm geleiteten Arbeiten sind in mehr als 250 Publikationen niedergelegt worden.

Cyril Grob war alles andere als ein Blender, er überzeugte vielmehr durch seinen sachlichen und auf soliden Fakten beruhenden Vortrag. Er war frei von jedem Geltungsdrang und eher von zurückhaltendem Charakter. Entsprechend pflegte er den gesellschaftlichen Kontakt mit Kollegen, Mitarbeitern und Studierenden eher spärlich. Sein Interesse war stets primär auf die Belange seiner Wissenschaft ausgerichtet gewesen.

Peter Schiess 